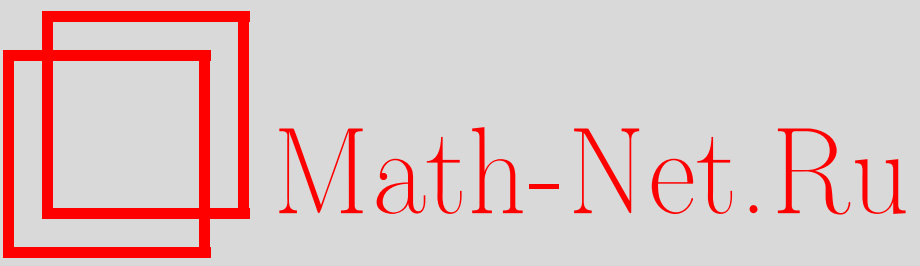

И. В. Изместьев, Многообразия, определяемые простыми многогранниками, как конфигурационные пространства шарнирных механизмов, УМН, 2000, том 55, выпуск 1, 185-186

DOI: https://doi.org/10.4213/rm256

Использование Общероссийского математического портала Math-Net.Ru подразумевает, что вы прочитали и согласны с пользовательским соглашением

http://www. mathnet.ru/rus/agreement

Параметры загрузки:

IP : 35.173 .137 .237

26 апреля 2023 г., 11:17:29 


\title{
МНОГООБРАЗИЯ, ОПРЕДЕЛЯЕМЫЕ ПРОСТЫМИ МНОГОГРАННИКАМИ, КАК КОНФИГУРАЦИОННЫЕ ПРОСТРАНСТВА ШАРНИРНЫХ МЕХАНИЗМОВ
}

\author{
И.В. ИЗМЕСТЬЕВ
}

В настоящей заметке строится шарнирный механизм, конфигурационное пространство которого эквивариантно гомеоморфно объединению нескольких экземпляров многообразия $\mathscr{Z}_{P}$, сопоставляемого всякому простому выпуклому многограннику $P$ (см. [1] и [2]).

ОПРЕДЕЛЕНИЕ 1 . Пусть $P$ - простой выпуклый многогранник размерности $n$ с $m$ гипергранями, $G$ - одна из групп $\mathbb{Z}_{2}$ или $S^{1}$. Пусть также фиксирована биекция между гипергранями многогранника $P$ и слагаемыми разложения $G^{m}=G \oplus G \oplus \cdots \oplus G$. Любой точке $p \in P$ соответствует подгруппа $H(p) \subset G^{m}$, порожденная группами всех тех гиперграней, которые содержат точку $p$. Тогда многообразие $\mathscr{Z}_{P}$ задается формулой $\mathscr{Z}_{P} \approx G^{m} \times P / \sim$, где $(g, p) \sim(h, q) \Leftrightarrow p=q$ и $g h^{-1} \in H(p)$.

ОПРЕДЕЛЕниЕ 2 ([3], см. также [4]-[6]). Шарнирным механизмом в $\mathbb{R}^{n}$ называется четверка $\mathscr{L}=(L, \ell, V, \mu)$, включающая (1) конечный граф̆ $L$ с множеством вершин $\mathcal{V}$ и множеством ребер $\mathscr{E} ;(2)$ отображение $\ell: \mathscr{E} \rightarrow(0,+\infty)$, сопоставляющее каждому ребру граффа $L$ положительное число, называемое его длиной; (3) подмножество $V \subset \mathscr{V}$ в множестве вершин графа $L$, элементы которого назьваются закрепленными вершинами; (4) отображение $\mu: V \rightarrow \mathbb{R}^{n}$. Шарнирный механизм в $\mathbb{R}^{2}$ назьвают плоским шарнирным механизмом.

Конфигурационное пространство $\mathscr{C}(\mathscr{L})$ шарнирного механизма $\mathscr{L}$ - это множество отображений $\phi: \mathscr{V} \rightarrow \mathbb{R}^{n}$ (так называемых реализаций механизма) со следующими свойствами: (1) $\left.\phi\right|_{V}=\mu ;(2)|\phi(v)-\phi(w)|=\ell(v, w)$ для любого ребра $(v, w) \in \mathscr{E}$. Полуконфигурационное пространство $\mathscr{S} \mathscr{C}(\mathscr{L}, \mathscr{W})$ состоит из сужений всех реализаций на некоторое подмножество $\mathscr{W} \subset \mathscr{V}$

Мы проведем рассуждение в случае $G=\mathbb{Z}_{2}$, для $G=S^{1}$ см. замечание 3 в конце статьи. Идея, позволяющая ввести на конфигурационном пространстве шарнирного механизма действие группы $\mathbb{Z}_{2}^{m}$, состоит в следующем. Пусть $v$-вершина графа $L$, имеющая степень 2 . Тогда в любой реализации $\phi$ механизма $\mathscr{L}$ образ вершины $v$ можно отразить симметрично относительно прямой, проходящей через образы смежных с ней вершин $v^{\prime}$ и $v^{\prime \prime}$, снова получив реализацию механизма. Если дан набор $\left\{v_{i}\right\}_{1}^{m}$ попарно несмежных вершин степени 2 , то инволюции, заданные этими вершинами, перестановочны и определяют действие $\mathbb{Z}_{2}^{m}$ на $\mathscr{C}$. Пространство орбит этого действия гомеоморфно полуконфигурационному пространству $\mathscr{S}=\mathscr{S} \mathscr{C}(\mathscr{L}, \mathscr{W})$, где $\mathscr{W}=\mathscr{V} \backslash\left\{v_{i}\right\}_{1}^{m}$. При этом $\mathscr{C} \approx \mathbb{Z}_{2}^{m} \times \mathscr{S} / \sim$, где $\left.\left(g,\left.\phi\right|_{\mathscr{W}}\right) \sim\left(h,\left.\psi\right|_{\mathscr{W}}\right) \Leftrightarrow \phi\right|_{\mathscr{W}}=\left.\psi\right|_{\mathscr{W}}$ и $g h^{-1} \in H(\phi), H(\phi)$ - группа инволюций, отвечающих тем из вершин $v_{i}$, для которых $\left|\phi\left(v_{i}^{\prime}\right)-\phi\left(v_{i}^{\prime \prime}\right)\right|=\ell\left(v_{i}, v_{i}^{\prime}\right)+$ $\ell\left(v_{i}, v_{i}^{\prime \prime}\right)$. Сравнивая с формулой из определения 1 , видим, что гомеоморфизм $\mathscr{C} \approx \mathscr{Z}_{P}$ обеспечивается гомеоморфизмом $\mathscr{S} \approx P$, сохраняющим соответствующие стационарные подгруппы: если $\left.\phi\right|_{\mathscr{W}} \mapsto p$, то $H(\phi)=H(p)$.

Здесь мы построим шарнирный механизм, конфигурационное пространство которого состоит из нескольких копий многообразия $\mathscr{Z}_{P}$. Мы следуем по пути, проложенному Кингом [3]-[5].

ТЕОремА. Для любого простого выпуклого многогранника $P$ существует плоский иарнирный механизм $\mathscr{L}$, конфигурационное пространство которого әквивариантно гомеоморфно несвязному обвединению нескольких әкземпляров многообразия $\mathscr{Z}_{P}$. Действие группь $\mathbb{Z}_{2}^{m}$ на пространстве $\mathscr{C}(\mathscr{L})$ осуществляется по описанному выше образиу.

ДоКАЗАТЕльСТво. Возьмем для начала $n$ подвижных вершин $w_{i}, i=1, \ldots, n$, каждая из которых соединена ребром длины $R$ с закрепленной вершиной, имеющей координаты $(0, R)$, где $R$ - достаточно большое число. Координаты точки $w_{i}$ вблизи нуля записываются как $\left(x_{i}, O(1 / R)\right)$ (символ отображения $\phi$ мы опускаем и под координатами точки $w_{i}$ понимаем координаты ее образа).

Важным элементом нашей конструкции будет пантограф (см. рис.), позволяющий производить деление отрезка в данном отношении. Если выполнены равенства $\overrightarrow{a d} / \overrightarrow{d e}=\overrightarrow{e f} / \overrightarrow{f b}=\lambda$, 


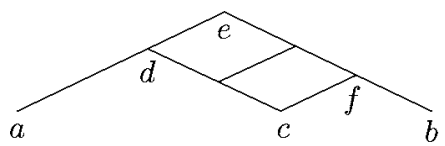

$d c=e f, c f=d e$, то имеем при любом положении точек $a$ и $b \overrightarrow{a c} / \overrightarrow{c b}=\lambda$. Вершины $a$ и $b$ будем называть исходными, вершину $c$ - функциональной, $\lambda$ - параметром пантограффа. Вершина $d$ закреплена во внутренней точке ребра $a е$ при помощи ребер $d a$ и $d e$ с суммарной длиной равной длине $а е$. Так же мы поступаем в других подобных случаях. Нетрудно, используя $n-1$ пантограф, вычислить значение любой линейной функции $\Lambda$ от переменных $x_{i}$, удовлетворяющей условию $\Lambda(1, \ldots, 1)=1$. Для этого нужно точки $w_{1}$ и $w_{2}$ сделать исходными вершинами первого пантограффа и для каждого следующего пантографа помешать одну из его исходных вершин в функциональную вершину предыдущего, а другую - в очередную из $w_{i}$. Функциональная вершина $t$ последнего пантограффа будет иметь координаты $(\Lambda(x), O(1 / R))$.

Рассмотрим теперь систему линейных неравенств, задающую многогранник $P$. Линейной заменой координат в $\mathbb{R}^{n}$ и умножением данных неравенств на константы приведем еек виду $\Lambda_{i}(x) \geqslant$ $-r_{i}\left(\leqslant r_{i}\right), i=1, \ldots, m$, где $\Lambda_{i}(1, \ldots, 1)=1, r_{i}>0$. Построим для каждого $i$ вершину $t_{i}$, $i=1, \ldots, m$, вычисляющую функционал $\Lambda_{i}$, и присоединим к ней ребром длины $\left(R+r_{i}\right) / 2$ вершину $v_{i}$, которую, в свою очередь, соединим ребром той же длины с вершиной, закрепленной в одной из точек $( \pm R, 0)$ - в зависимости от знака соответствующего неравенства. При любой реализации механизма расстояние между вершинами $( \pm R, 0)$ и $t_{i}$ не превосходит $R+r_{i}$, и это условие после несложных преобразований можно записать в виде $\Lambda_{i}(x) \geqslant-r_{i}+O\left(1 / R^{3}\right)$ $\left(\leqslant r_{i}+O\left(1 / R^{3}\right)\right)$ при достаточно большом $R$. Кроме того, при большом $R$ эти неравенства являются единственными ограничениями на $x$-координаты точек $w_{i}$, естественно интерпретируемыте как координаты в полуконфигурационном пространстве $\mathscr{S} \mathscr{C}\left(\mathscr{L},\left\{w_{i}\right\}\right)$, которое, таким образом, оказьвается гомеоморфно многограннику $P$ с сохранением стационарных подгрупп (ввиду простоты $P$ добавочные члены $O\left(1 / R^{3}\right)$ при большом $R$ не изменяют гранной структуры). Вследствие этого полуконфигурационное пространство $\mathscr{S} \mathscr{C}\left(\mathscr{L},\left\{w_{i}\right\} \cup\left\{v_{i}\right\}\right)$ эквивариантно гомеоморфно многообразию $\mathscr{Z}_{P}$. Все конфигурационное пространство $\mathscr{C}(\mathscr{L})$ будет набором нескольких копий $\mathscr{Z}_{P}$ ввиду наличия у каждого пантографо симметрии, сохраняющей исходные и функциональную вершины. Доказательство окончено.

ЗАмечАния. 1) Шарнирный механизм позволяет получить эквивариантное вложение многообразия $\mathscr{L}_{P}$ в евклидово пространство $\mathbb{R}^{n+m}$ с ортогональным действием группы $\mathbb{Z}_{2}^{m}$. Координаты точки, соответствующей данной реализации, это $\left\{x_{i}\right\}_{1}^{n}$ и ориентированные площади треугольников с вершинами $( \pm R, 0), v_{i}, t_{i}$ (которые меняют свой знак под действием элементов группы $\left.\mathbb{Z}_{2}^{m}\right)$. 2) Построенный механизм содержит $O(\mathrm{~nm})$ ребер и вершин. Это количество можно уменьшить до $O(n \ln m)$, если соединять пантограффы не последовательно, а в виде двоичного дерева. 3) Для случая действия тора $\left(S^{1}\right)^{m}$ аналогичный шарнирный механизм строится в $\mathbb{R}^{3}$ : заметим, что в трехмерной реализации на вершину степени 2 действует окружность точно так же, как в плоской реализации действовала группа $\mathbb{Z}_{2}$.

Автор выражает благодарность профессору В. М. Бухштаберу и профессору А.Б. Сосинскому за стимулирующие обсуждения и любезно предоставленный материал.

\section{СПИСОК ЛИТЕРАТУРЫ}

[1] Davis M.W., Januszkiewicz T. // Duke Math. J. 1991. V. 62. № 2. P. 417-451. [2] Бухштабер В. М., Панов Т. Е. // Тр. МИАН. 1999. Т. 225. C. 96-131. [3] King H. С. Semiconfiguration spaces of planar linkages // Preprint, math.GT/9810130. [4] King H. C. Planar linkages and algebraic sets // Preprint, math.AG/9807023. [5] King H.C. Configuration spaces of linkages in $R^{n}$ // Preprint, math.GT/9811138. [6] Звонкин Д. // Russian J. Math. Phys. 1996. T. 5. № 2. C. 247-266. 